\title{
Caracterização no Infravermelho (IV) e Eletrônica de superfície (MEV) de membranas assimétricas à base de Poli (acrilonitrila-co-acetato de vinila)
}

\author{
Characterization Infrared (IR) and Surface \\ electron microscopy (SEM) of asymmetric \\ membranes based on Poly (acrylonitrile-co-vinyl acetate)
}

\author{
Paulo César de Faria ${ }^{1}$, Airton Abrahao Martin ${ }^{2}$, \\ Nilton Pereira Alves ${ }^{3}$
}

\footnotetext{
1,2 Laboratório de Espectroscopia Vibracional Biomédica - LEVB/IP\&D/ UNIVAP CEP: 12244-000, São José dos Campos, SP.

e-mail: lacfaria@gmail.com;amartin@univap.br

${ }^{3}$ Quimlab Química \& metrologia. Rodovia Geraldo Scavone, 2.300 - Jardim Califórnia CEP: 12.305-490 / Jacareí, SP. e-mail: nilton@quimlab.com.br
}

\begin{abstract}
RESUMO
As membranas assimétricas, denominadas respectivamente $\mathrm{Mb} 1$ e Mb2, foram obtidas por meio do método de inversão de fases, empregando-se o copolímero Poli (acrilonitrila-co-acetato de vinila) - P (AN-co-AV) com composição em massa inicial de $6,5 \%$ e $8,5 \%$ de Polivinilpirrolidona (PVP). As membranas foram caracterizadas por meio das técnicas de Espectroscopia no Infravermelho com Transformada de Fourier (IVTF) e de Microscopia Eletrônica de Varredura (MEV). Por meio da técnica de IV-TF foi possível identificar as bandas que permitiram apontar os principais grupos funcionais presentes: i) no polímero base, ii) no copolímero e iii) no aditivo polimérico PVP usados na fabricação das membranas. Tais bandas seriam: em 2246 $\mathrm{cm}^{-1}$, banda associada à deformação axial do grupo $\mathrm{C} \equiv \mathrm{N}$ da unidade de acrilonitrila; em $1735 \mathrm{~cm}^{-1}$, banda associada ao estiramento do grupo carbonila $(\mathrm{C}=\mathrm{O})$, presente no comonômero éster ou ácidos utilizados na polimerização da Poliacrilonitrila (PAN), e em $1242 \mathrm{~cm}^{-1}\left(v_{\mathrm{C}-\mathrm{O}}\right)$, banda associada aos grupos acetatos presentes no copolímero $\mathrm{P}(\mathrm{AN}-\mathrm{co}-\mathrm{AV})$. As bandas fundamentais da PVP foram identificadas em $1664 \mathrm{~cm}^{-1}$ e 1293 $\mathrm{cm}^{-1}$ atribuída ao estiramento do grupo carbonila $(\mathrm{C}=\mathrm{O})$ ligado à amida e ao estiramento da ligação $(-\mathrm{C}-\mathrm{N})$ respectivamente. Os resultados obtidos por meio da MEV apontaram para alterações estruturais na morfologia da membrana resultantes do aumento na concentração de PVP.
\end{abstract}

Palavras chaves: Membranas Assimétricas, Poliacrilonitrila, Polivinilpirrolidona, Infravermelho e Microscopia Eletrônica de Varredura.

\begin{abstract}
Asymmetric membranes, denominated respectively Mb1 and MB2, were obtained by the phase inversion method, employing the copolymer poly (acrylonitrile-co- vinyl acetate) - P (AN-co-VA) with an initial weight of composition $6.5 \%$ and $8.5 \%$ Polyvinylpyrrolidone (PVP). Membranes were characterized by means of spectroscopy techniques with Infrared Fourier Transform (FT-IR) and Scanning Electron Microscopy (SEM). Through the FT-IR technique it was possible to identify the bands that allowed showing the main functional groups present: i) in the base polymer ii) in the copolymer and iii) in the PVP polymeric additive used in the manufacture of membranes. Such bands are: in $2246 \mathrm{in}^{-1}$, band associated with the axial deformation of the $\mathrm{C} \equiv \mathrm{N}$ group of acrylonitrile unit; in $1744 \mathrm{~cm}^{-1}$, band associated with stretching of the carbonyl group $(\mathrm{C}=\mathrm{O})$, present in the comonomer ester or acid used in the polymerization of Polyacrylonitrile (PAN), and in $1242 \mathrm{~cm}^{-1}$ ( $\left.\mathrm{vC}-\mathrm{O}\right)$, band associated with the acetate groups present in copolymer $\mathrm{P}(\mathrm{AN}-\mathrm{co}-$ VA). The fundamental bands of PVP were identified in $1664 \mathrm{~cm}^{-1}$ and in $1293 \mathrm{~cm}^{-1}$ attributed to the stretching of the carbonyl group $(\mathrm{C}=\mathrm{O})$ attached to the amide and the stretching of the bond $(-\mathrm{C}-\mathrm{N})$, respectively. The results obtained by SEM pointed to structural changes in membrane morphology resulting from the in-
\end{abstract}


crease in PVP concentration.

Keywords: Asymmetric Membrane, Polyacrylonitrile, polyvinylpyrrolidone, FT-IR and SEM.

\section{INTRODUÇÃO}

Para se fabricar membranas sintéticas, empregadas nos processos de separação por membranas (PSM), três aspectos são de fundamental importância: i) a seleção do material, ii) as condições de preparo, as quais determinam a morfologia e, além disso, são fundamentais para aperfeiçoar as propriedades de transporte, e finalmente iii) a configuração do módulo [1].

O copolímero Poli (acrilonitrila-co-acetato de vinila) - P(AN-co-AV) escolhido e empregado na fabricação de membranas é amplamente empregado na produção de fibras acrílicas para a indústria têxtil [2] devido a influencia que o comonômero neutro acetato de vinila (AV), presente poliacrilonitrila (PAN), exerce na solubilização desta diante de seus solventes orgânicos. Portanto, determinada concentração de AV atribui ao copolímero qualidade essencial à fiação, proporcionando melhor capacidade de estiragem ou alongamento das fibras [3].

A capacidade de solubilização dos materiais é determinada pela polaridade desses. Portanto, polímeros apolares, como as polissulfonas [4], são solúveis em solventes apolares, e polímeros polares, como os derivados de celulose [5], são dissolvidos em solventes polares. A Poliacrilonitrila (PAN), que apresenta certa dificuldade em solubilizar-se, é dissolvida somente em solventes polares, tais como: a N-metil-2pirrolidona (NMP) [6], a Dimetilacetamida (DMAc) [7] e a Dimetilformamida (DMF) [8]. A DMF é o mais empregado, proporcionando excelente solubilidade a PAN e a seus compósitos [9].

As membranas poliméricas são amplamente preparadas pela técnica da inversão de fases (IF) [10]. Esse processo inicia-se com um desequilíbrio da solução polimérica, atribuído à indução do estado de supersaturação na presença de um não-solvente (água), ou até mesmo às variações de temperatura da solução, o que a torna instável, e causa sua separação em duas fases [9,11]: uma fase sólida, rica em polímero, a qual forma a matriz da membrana propriamente dita; e uma fase líquida, pobre em polímero, a qual forma a estrutura porosa. Na fabricação de membranas, a solução é espalhada sobre um suporte poroso e/ou rígido e liso formando um filme fino de espessura uniforme, em seguida é conduzida ao banho de não-solvente. A IF ocorre devido a uma troca (inversão) entre o solvente presente no filme e o não-solvente presente no banho, induzindo à precipitação do solvente [1].

As membranas são constituídas de diferentes características morfológicas, podendo ser densas ou porosas. As membranas do tipo porosas apresentam poros semelhantes a um cone, cujo diâmetro aumenta de forma gradual em sua seção transversal [12]. A região superior da membrana (2\% de sua espessura global), que está em contato com a solução a ser separada, pode ou não apresentar poros muito pequenos $(<0,05 \mu \mathrm{m})$, o que interfere na seletividade [1].

O aditivo polimérico poli (vinil pirrolidona) - PVP, empregado na obtenção e controle do diâmetro de poros [9], possui excelente solubilidade em água e favorece a fase liquida (pobre em polímero), promovendo estruturas morfologicamente porosas [7]. Membranas preparadas a partir da PAN e do aditivo PVP no controle de porosidade foram utilizadas em tratamento de águas residuais por meio da ultrafiltração (UF). Nestes trabalhos, os pesquisadores [13] constataram aumento no desempenho (funcionalidade) das membranas quando aumenta a massa da PVP. Existem outras medidas adotadas no controle do diâmetro dos poros tais como: (i) variação da temperatura durante a fusão da solução polimérica, (ii) composição (\% em massa dos componentes) da solução polimérica [14], (iii) composição do banho na técnica de precipitação por imersão (ou o tipo de não-solvente utilizado); e o (iv) aquecimento do banho de não-solvente (variação da temperatura) [6].

Conforme exposto anteriormente, o presente estudo tem por objetivo investigar por meio da técnica de IV-TF as bandas que apontam os principais grupos funcional presentes: no polímero base (PAN), no copolímero - P(AN-co-AV) e no aditivo polimérico (PVP) usados na fabricação das membranas. E ainda, caracterizar por meio da Microscopia Eletrônica de Varredura (MEV) os efeitos provocados pela adição da PVP (de $6,5 \%$ para $8,5 \%$ ) em massa, na formação das membranas.

\section{MATERIAIS E MÉTODOS}

\subsection{Material e polímeros utilizados}

Os seguintes materiais foram empregados no preparo das membranas: o copolímero poli (acrilonitrila-co- 
acetato de vinila) - $\mathrm{P}(\mathrm{AN}-\mathrm{co}-\mathrm{AV})$, sendo $94 \%$ de poliacrilonitrila (massa molecular média numérica -- $\mathrm{M}_{\mathrm{n}}-$ de aproximadamente $33.000 \mathrm{~g} / \mathrm{mol}$ ), fornecido pela Quimlab Química; o aditivo poli (vinil pirrolidona) - PVP (K-30 com $\mathrm{M}_{\mathrm{n}}$ de $30.600 \mathrm{~g} / \mathrm{mol}$ ), do fornecedor Synth; o solvente Dimetilformamida - DMF (> 99\%), de procedência BASF; e Álcool Etílico e n-Hexano de purezas PA/ACS, ambos do fornecedor Vetec.

\subsection{Fabricação de membranas poliméricas por inversão de fase}

Foram empregadas duas concentrações mássicas diferentes para a PVP na solução polimérica $\mathrm{P}(\mathrm{AN}-\mathrm{co}-\mathrm{AV})$ + PVP + DMF, fixando-se o mesmo valor em massa para a PAN em ambos os casos. Assim, a membrana obtida a partir da composição em massa inicial da solução polimérica de $\mathrm{P}(\mathrm{AN}-$ co-AV) / PVP / DMF (12,5 / $6,5 / 81,0 \% \mathrm{~m} / \mathrm{m}$ ) foi denominada de $\mathrm{Mb} 1$, enquanto a membrana $\mathrm{Mb} 2$ foi preparada com uma concentração maior de PVP na solução polimérica $(12,5 / 8,5 / 79,0 \% \mathrm{~m} / \mathrm{m})$. As duas soluções poliméricas foram preparadas em Becker de vidro, misturando-se o copolímero, o aditivo e o solvente com o auxílio de um bastão de vidro, e em seguida aquecendo-se a mistura a aproximadamente $65^{\circ} \mathrm{C} \pm 5^{\circ} \mathrm{C}$ até dissolver todos os componentes. A massa de cada solução polimérica foi de aproximadamente $10,0 \mathrm{~g}$. A solução polimérica foi espalhada em placas lisas de vidro com área de 20 x $20 \mathrm{~cm}$. Em seguida, as placas de vidro com a solução polimérica espalhada foram submetidas a um banho em cuba de vidro contendo água para precipitação (coagulação) da $\mathrm{P}(\mathrm{AN}-\mathrm{co}-\mathrm{AV})$, levando à formação da membrana e também permitindo a solubilização da PVP para a formação de poros. Os filmes permaneceram imersos no banho contendo o não-solvente por 10 minutos. Após o banho de precipitação, as membranas passaram por um processo de desidratação e eliminação do restante da DMF por meio da técnica de troca de solvente. As membranas permaneceram por mais 10 minutos em etanol anidro dentro de uma cuba de vidro e por mais 10 minutos em n-hexano, também em cuba de vidro. Após esta permanência, as membranas foram alocadas entre superfícies porosas à temperatura ambiente, por 72 horas, para completar o processo de secagem. A membrana obtida a partir da solução polimérica apresentou espessura media de aproximadamente $0,75 \mathrm{~mm}$.

\subsection{Caracterização das membranas}

As membranas $\mathrm{Mb} 1$ e Mb2 foram caracterizadas por meio da técnica de Espectroscopia no Infravermelho com Transformada de Fourier (IV-TF) com o intuito de se investigar os principais grupos funcionais presentes nestas membranas e, em seguida, se analisar os efeitos que a concentração em massa da PVP causou na formação dos poros das membranas por meio da Microscopia Eletrônica de Varredura (MEV).

\subsubsection{Espectroscopia no Infravermelho com transformada de Fourier (IV-TF)}

Empregou-se a técnica de análise por infravermelho para identificar os principais grupos químicos presentes nas membranas P(AN-co-AV) / PVP / DMF (Mb1 e Mb2). Foram preparadas pastilhas de brometo de potássio - $(\mathrm{KBr})$, utilizando-se pastilhador da marca PIKE de $13 \mathrm{~mm}$ de diâmetro em prensa hidráulica de 10 ton. Cada pastilha foi produzida macerando-se $1,5 \mathrm{mg}$ da amostra (filmes poliméricos) com $130 \mathrm{mg}$ de $\mathrm{KBr}$ em almofariz de porcelana. A mistura de $\mathrm{KBr}$ foi prensada no pastilhador por 10 minutos. A pastilha obtida foi analisada em um espectrofotômetro Varian Modelo IR-640 em modo de transmitância na região de 4000 a $600 \mathrm{~cm}^{-1}$.

\subsubsection{Microscopia Eletrônica de Varredura (MEV)}

$\mathrm{O}$ aspecto morfológico das membranas foi verificado por meio da técnica de Microscopia Eletrônica de Varredura (MEV). Para tanto, as membranas foram imersas em nitrogênio líquido e fraturadas criogenicamente, em seguida, foi aplicado um tratamento térmico a $55^{\circ} \mathrm{C}$ durante 24 horas. As amostras foram metalizadas em solução coloidal de ouro em um metalizador modelo K550X da EMITECH.

Todas as membranas foram caracterizadas em um microscópio eletrônico de varredura (MEV - EVO) para obter informações topográficas da morfologia de suas superfícies superior e inferior e de sua seção transversal. As micrografias foram processadas utilizando-se um analisador de imagens (Zeiss). 


\section{RESULTADOS E DISCUSSÃO}

\subsection{Espectroscopia no Infravermelho com Transformada de Fourier (IV-TF)}

A Figura 1 monstra o espectro do copolímero de $\mathrm{P}(\mathrm{AN}-\mathrm{co}-\mathrm{AV})$. As principais bandas que identificam este copolímero são as bandas em $2246 \mathrm{~cm}^{-1}$ associada à deformação axial do grupo $\mathrm{C} \equiv \mathrm{N}$ da unidade monomérica de acrilonitrila, em $2940 \mathrm{~cm}^{-1}\left(v_{\mathrm{C}-\mathrm{H}} \mathrm{em} \mathrm{CH} \mathrm{CH}_{2}\right)$, em $1452 \mathrm{~cm}^{-1}\left(\delta_{\mathrm{C}-\mathrm{H}} \mathrm{em} \mathrm{CH}_{2}\right)$ e em $1367 \mathrm{~cm}^{-1}\left(\delta_{\mathrm{C}-\mathrm{H}} \mathrm{em} \mathrm{CH}\right)$, sendo essas três ultimas bandas características dos grupos $\mathrm{CH}$ das cadeias acíclicas alifáticas ao longo da cadeia principal $[13,15]$.

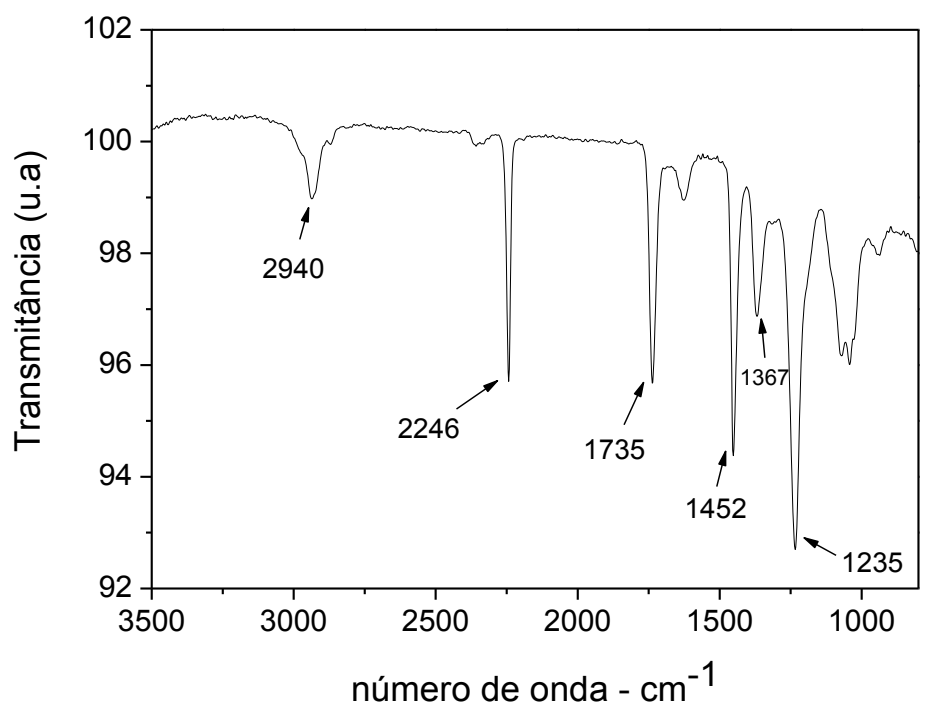

Figura 1: Espectro do copolímero Poli (acrilonitrila-co-acetato de vinila) - P(AN-co-AV).

A intensa banda que aparece em $1735 \mathrm{~cm}^{-1}$ é uma banda característica de estiramento do grupo carbonila $(\mathrm{C}=\mathrm{O})$. Essas bandas aparecem devido ao monômero éster ou ácido, ambos utilizados durante a polimerização da PAN [16]. Além da banda da carbonila, ocorre uma intensa banda em $1235 \mathrm{~cm}^{-1}\left(v_{\mathrm{C}-0}\right)$, banda característica de grupos acetatos, comprovando a existência da unidade de acetato de vinila no copolímero de PAN.

Por meio da figura 2, foi possível estabelecer os grupos químicos que caracterizam o aditivo polimérico poli (vinil pirrolidona) - PVP: o grupo carbonila $(\mathrm{C}=\mathrm{O})$ ligado à amida na cadeia cíclica de carbonos [17, 18] e o grupo nitrila $\mathrm{C}-\mathrm{N}\left(v_{\mathrm{C}-\mathrm{N}}\right)$ situado entre a cadeia cíclica e a cadeia linear de carbonos [19, 18]. O grupo carbonila foi identificado por meio da banda $1664 \mathrm{~cm}^{-1}$, associada ao estiramento desse grupo. E o grupo nitrila foi identificado por meio da banda $1293 \mathrm{~cm}^{-1}$ associada ao estiramento da ligação desse grupo. A larga banda em torno de $3429 \mathrm{~cm}^{-1}$ expressa a vibração do estiramento $\mathrm{O}-\mathrm{H}$ na PVP devido à presença de água neste polímero por ser altamente higroscópico. A banda em $2954 \mathrm{~cm}^{-1}$ surge a partir do estiramento do $-\mathrm{CH}_{2}$. Observa-se também as frequências vibracionais das bandas em $1497 \mathrm{~cm}^{-1}$, associada à deformação do $-\mathrm{CH}_{2}$ $\left(\delta_{\mathrm{CH} 2}\right)$ da cadeia linear do polímero poli (vinil pirrolidona), e em $1439 \mathrm{~cm}^{-1}$, associada à deformação assimétrica do $\mathrm{CH}\left(\delta_{\mathrm{C}-\mathrm{H}}\right)$ no $\mathrm{CH}_{2}$ - da cadeia cíclica do PVP. 


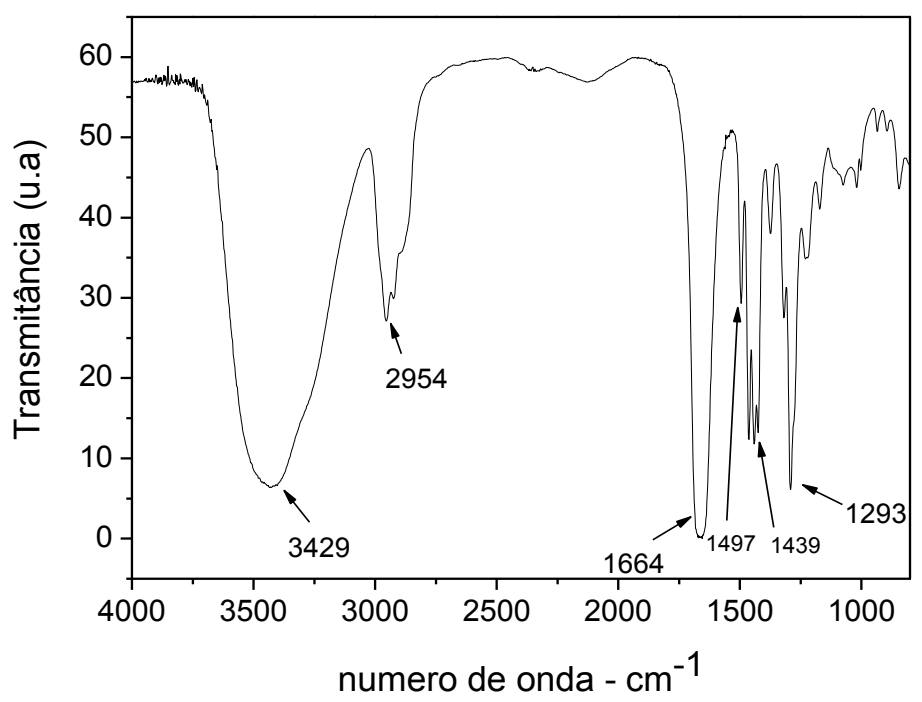

Figura 2: Espectro de IV-TF da Poli (vinil pirrolidona) - PVP.

Os materiais poliméricos utilizados na fabricação das membranas foram o copolímero $\mathrm{P}(\mathrm{AN}-\mathrm{co}$-AV) e $\mathrm{o}$ aditivo PVP. Ambos os materiais apresentam bandas que permitem identificar sua presença nas membranas. Por exemplo, as bandas do copolímero $\mathrm{P}(\mathrm{AN}-\mathrm{co}-\mathrm{AV})$ são identificadas em $2246 \mathrm{~cm}^{-1}$, banda associada ao grupo $\mathrm{C} \equiv \mathrm{N}$ da acrilonitrila, e em $1235 \mathrm{~cm}^{-1}$, banda associada ao comonômero acetato de vinila (AV). E as bandas que caracterizam a PVP são encontradas em $1664 \mathrm{~cm}^{-1}$, banda associada ao grupo carbonila $(\mathrm{C}=\mathrm{O})$, e em $1293 \mathrm{~cm}^{-1}$, associada ao alongamento da ligação (-C-N) [20, 21]. Assim sendo, essas bandas estão presentes nos espectros das membranas Mb1 e Mb2 (Fig. 3). Em outros trabalhos, que se tem por estudo a análise da PVP, os autores descrevem o estiramento da carbonila que se liga à amida da cadeia cíclica [17]. Portanto, observam-se que a presença deste aditivo polimérico (PVP) apresenta as bandas características de seu grupo funcional $(\mathrm{C}=\mathrm{O})$, tanto na membrana $\mathrm{Mb} 1$ quanto na membrana $\mathrm{Mb}$ 2, preparadas pelo método de inversão de fase. Assim sendo, há uma evidência plausível de que a PVP permaneceu nas membranas após o banho [11].

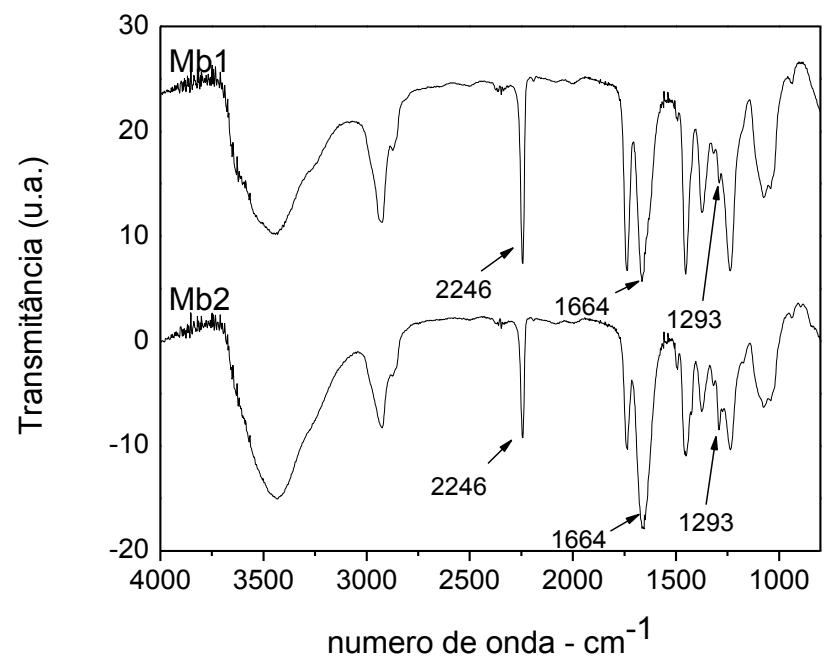

Figura 3: Espectros das membranas Mb1 e Mb2 - P(AN-co-AV), DMF e PVP (de 6,5\% para 8,5\%).

\subsection{Morfologia das membranas via Microscopia eletrônica de varredura (MEV)}

Um dos parâmetros investigativos utilizados na caracterização das membranas poliméricas é a sua natureza morfológica, por exemplo, a distribuição e tamanho dos poros, a porosidade superficial a espessura, os quais 
constituem parâmetros morfológicos bastante relevantes. O principal motivo do êxito de uma membrana polimérica preparada pela técnica de IF, desenvolvida pelos métodos acadêmicos de Loeb e Sourirajan, está relacionado à sua morfologia singular, que apresenta poros gradualmente maiores em sua seção transversal. A região superior da membrana, a qual apresenta a "pele" de aproximadamente $2 \%$ de sua espessura (principal responsável pela seletividade desejada), pode não apresentar poros ou pode possuir poros muito pequenos $(<0,05 \mu \mathrm{m})$. A outra região, que segue logo abaixo da "pele", denominada suporte (substrato), tem como principal função fornecer resistência mecânica à "pele", que apresenta como característica canais porosos cujo diâmetro segue aumentando de maneira progressiva. As membranas que apresentam essas características morfológicas são denominadas anisotrópicas ou assimétricas [1].

Neste parágrafo, são apresentadas as características morfológicas das membranas Mb1 e Mb2, analisadas via MEV, a fim de investigar os aspectos gerais das três regiões que formam a membrana assimétrica: a superfície superior, onde se situa a "pele", a seção transversal, que apresenta poros progressivamente maiores conforme se aproximam da superfície inferior, e a superfície inferior, onde culmina o maior diâmetro dos poros. A Figura 4 expõe a superfície superior, região que exibe a "pele" da membrana $\mathrm{Mb} 1$, praticamente homogênea quando analisada em macro-escala. Tal região apresenta poros pouco definidos para o aumento de $10 \mu \mathrm{m}$. Neste plano (região), encontra-se a parte seletiva da membrana, parte essa formada durante a IF, responsável pela assimetria da membrana. O mecanismo que forma a membrana é iniciado por meio de uma indução de instabilidade termodinâmica, provavelmente provocada por mudanças na concentração ou temperatura das diferentes soluções. Tal instabilidade leva a solução a separar-se em pelo menos duas fases, uma fase rica e outra pobre em polímero. A fase rica em polímero dará origem à estrutura sólida da membrana, enquanto a fase pobre dará origem aos poros. Durante esse processo de separação entre as duas fases (rica e pobre), a concentração polimérica da fase rica aumenta significativamente, elevando sua viscosidade e dificultando sua transferência de massa no sistema, o que resulta na formação de duas estruturas diferentes: uma camada superior densa e uma subcamada porosa. A camada superior densa é formada devido ao aumento da viscosidade da solução polimérica, aumento esse decorrente de um dos seguintes fenômenos, o de vitrificação, o de gelificação ou o de cristalização [22, 23]. Desta maneira, a camada superior densa é a primeira a ser formada no processo de separação entre as fases (rica e pobre) e passa a ser considerado sólido. Após a formação da "pele" densa, a filtrante, ocorre à formação das outras regiões da membrana, processo que demanda alguns segundos, conforme a espessura do filme polimérico adquirido durante o seu espalhamento. Segundo os pesquisadores [24, 25], duas separações ocorrem entre a solução polimérica e o banho de coagulação, uma separação ocorre de maneira instantânea e a outra de forma mais lenta. As membranas formadas pelo mecanismo de separação instantâneo apresentam uma subcamada (seção transversal) altamente porosa e uma camada superior fina e densa, podendo ou não haver presença de poros. Portanto, quanto maior for a taxa de saída do solvente, mais a fase rica em polímero ficará concentrada durante a precipitação e, consequentemente, menos porosa será a região superior [26]. E no processo de separação lenta, as membranas resultantes apresentam estrutura com característica tipicamente esponjosa.

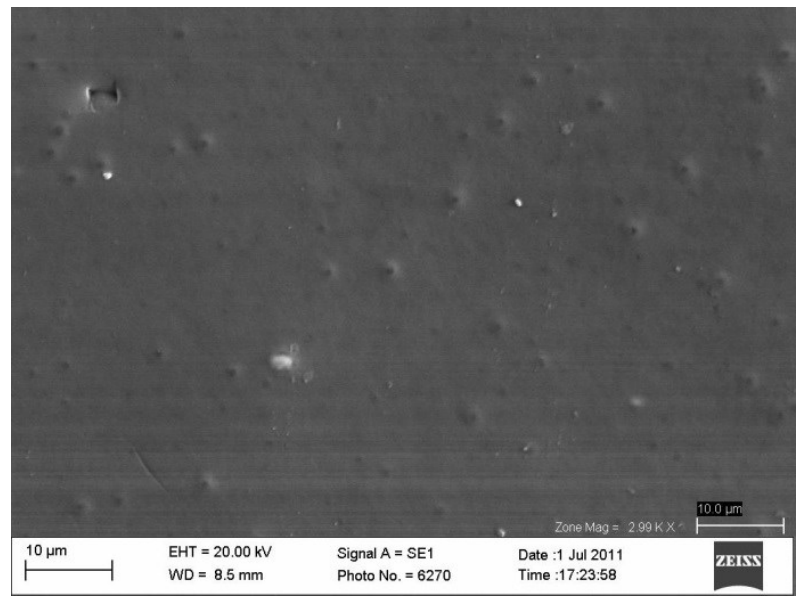

Figura 4: Superfície superior da membrana Mb1 (6,5\% de PVP).

Quanto ao aspecto da seção transversal da membrana Mb1 (6,5\% de PVP) Fig. 5, nota-se em macroescala espaços vazios e canais porosos desorganizados, ambos possivelmente resultantes da fratura criogêni- 
ca, e apesar da disposição irregular dos poros, é possível a visualização da pele densa delimitando a assimetria da membrana. A precipitação da membrana deve-se ao aumento na concentração de polímero [6, 27] e à diferença de potencial químico tanto do solvente na solução polimérica quanto da água do banho de coagulação. Assim, os primeiros núcleos são formados na camada logo abaixo da interface precipitada e crescem à custa do solvente, dando origem a esses espaços vazios que aparecem desarrumados e alargados, mas tendem a se alinhar, em formato de cilindros cônicos, ao longo da seção transversal quando se aumenta a concentração do polímero. Alterações podem ocorrer na estrutura morfológica e na hidrofilicidade das membranas, devido ao tipo de material polimérico utilizado na fabricação e também ao tipo de aditivo, neste caso, a PVP, utilizada por ser hidrossolúvel. Assim, a adição da PVP, aditivo pouco miscível com a solução polimérica e bastante hidrofílico, favorece o processo de separação instantânea e promove a formação de poros e a interconexão da superfície superior e inferior através dos canais porosos. Segundo os pesquisadores e os colaboradores [28], nos instantes iniciais em que ocorre a imersão da solução polimérica, dois de seus componentes, o polímero e o aditivo (PVP), se comportam como um só.

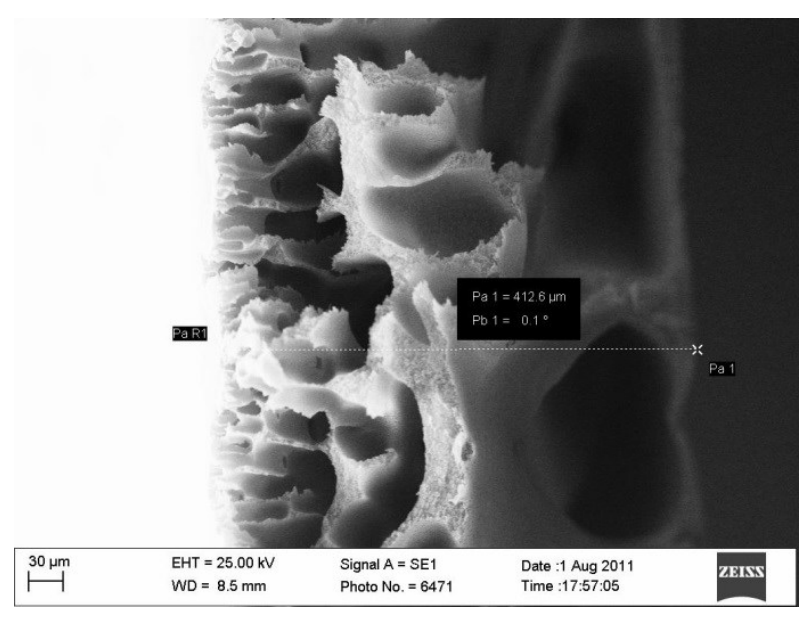

Figura 5: Seção transversal da membrana Mb1 (6,5\% de PVP).

A fotomicrografia da superfície inferior da membrana Mb1, apresentada na Fig. 6, mostra a presença de poros, sem uma forma geométrica definida (irregulares), por quase toda a sua superfície. A superfície porosa desta região da membrana foi obtida com a adição de $6,5 \%$ da PVP, resultando em uma densidade porosa de aproximadamente 380 poros em uma área de $477,47 \mu \mathrm{m}^{2}$, poros esses de diâmetros maiores e menores. Devido à formação dos poros de diâmetros diferentes, foi estabelecido um diâmetro médio aparente dos maiores poros, $1,33 \pm 0,2 \mu \mathrm{m}$, e o diâmetro médio aparente dos menores poros, $0,43 \pm 0,1 \mu \mathrm{m}$. De acordo com os pesquisadores [29], a formação dessa subcamada porosa é resultante da saída do solvente da solução polimérica para o banho de coagulação. Nesse momento, a água infiltra na solução polimérica formando os núcleos que correspondem à fase pobre em polímero. A partir da fase rica em polímero, esses núcleos tendem a crescer, originando os poros e as paredes que formam os canais porosos. Com a adição da PVP, acentuam-se as mudanças na estrutura morfológica, proporcionando ao material maior hidrofilicidade e interconexão das superfícies superior e inferior [28, 30] através dos canais porosos. 


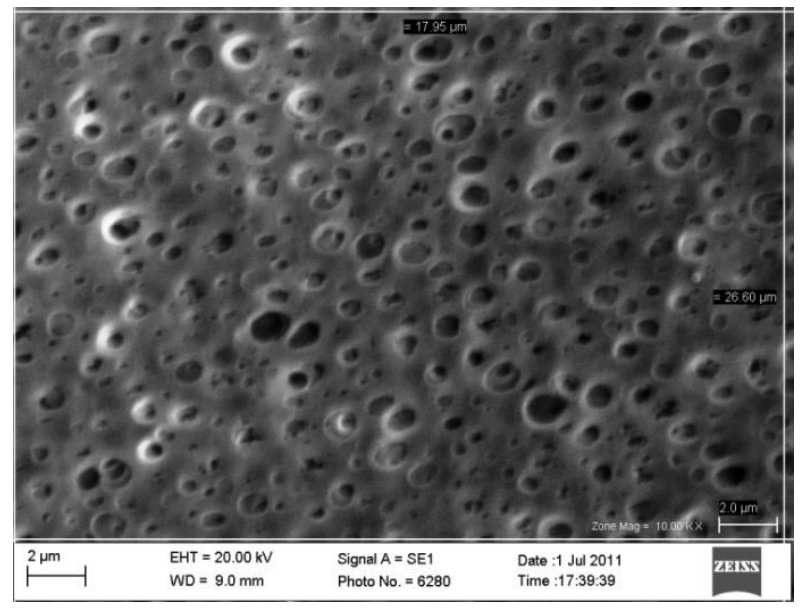

Figura 6: Superfície inferior da Mb1 (6,5\% de PVP).

A Figura 7 ilustra, em baixa definição, a superfície superior da membrana $\mathrm{Mb} 2$, a qual não apresenta uma regularidade se comparada à superfície superior da $\mathrm{Mb} 1$, o que provavelmente pode ser resultado do material utilizado durante o espalhamento da solução polimérica. Essa superfície apresenta considerável irregularidade, porém permanece com suas características homogêneas. Alguns fatores podem ser a causa da alteração desta superfície morfológica da membrana, tais como: a natureza do polímero, o tipo do solvente, a presença ou não de água e aditivos, as condições em que ocorre a precipitação ou até mesmo os procedimentos adotados durante a fabricação da membrana, a espessura de espalhamento do filme, o material usado no espalhamento, o tempo de exposição da solução polimérica ao ser espalhada e até mesmo a temperatura do banho de coagulação [1] .

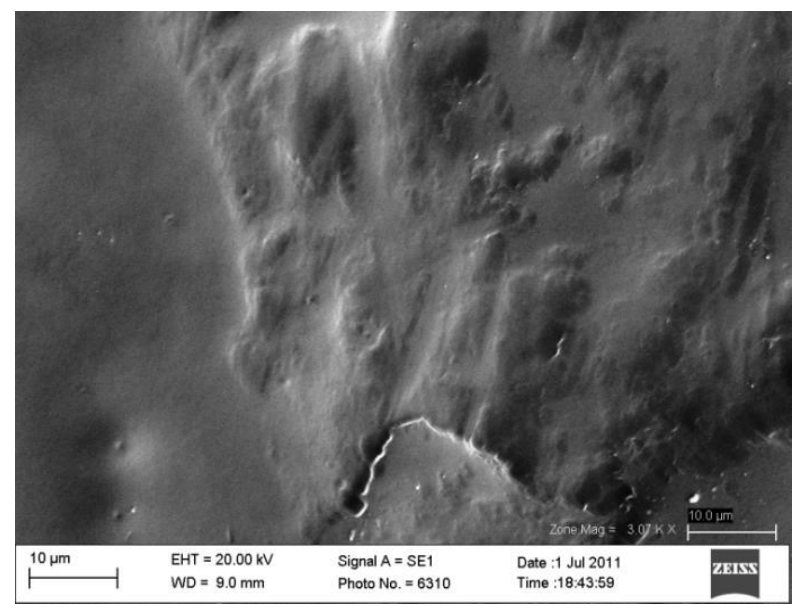

Figura 7: Superfície superior da membrana Mb2 (8,5\% de PVP).

Na Figura 8, seção transversal da membrana Mb2, observa-se que a membrana, após aumento na adição de PVP para 8,5\% em massa, apresenta significativa redução dos espaços vazios e estreitamento das paredes dos canais porosos, redução e estreitamento esses que resultaram na formação dos poros. Tais poros estão bem mais definidos, fato que se deve, além da alta resolução do microscópio, ao bom direcionamento dos canais porosos, os quais estão dispostos perpendicularmente em relação à "pele" densa da superfície superior da membrana. Com o aumento daPVP (de 6,5\% para 8,5\%), a morfologia assimétrica da membrana tornou-se mais evidente. Segundo pesquisas sobre [31] a adição de diferentes concentrações em massa de PVP e os efeitos dessa adição na estrutura morfológica das membranas assimétricas, a termodinâmica e a cinética constituem dois fatores determinantes que controlam a morfologia na inversão de fase. $\mathrm{O}$ aditivo polimérico PVP funciona como um agente sinérgico, que suprime a formação de espaços vazios (fator cinéti- 
co) quando aumenta a sua massa na solução, enquanto a baixa concentração do aditivo promove a formação destes espaços vazios (efeito termodinâmico). Sendo assim, estabelece-se uma competição entre o efeito termodinâmico e o cinético da PVP no processo de inversão de fase. Em outras pesquisas realizadas com a adição de diferentes concentrações em massa de PVP, os autores constatam que as membranas preparadas com esse aditivo apresentam uma camada superior densa, denominada "Top Layer", e uma subcamada macroporosa. Para a PVP (K-10), as alterações na morfologia foram quase imperceptíveis, entretanto para as PVP (k40 e K-360), ocorreram mudanças notáveis, em particular na PVP (K-360). Os autores relatam que existem vários estudos a respeito da solubilidade da PVP em água e da função que esse aditivo exerce, desempenhando um papel importante nos PSM [32] e também na obtenção de membranas assimétricas. Portanto, quando a PVP, que tem como característica ser hidrofílica, deixa a solução polimérica e une-se água, o aditivo promove um arraste interno que resulta na formação dos canais porosos e na diminuição do diâmetro dos poros, fazendo com que a membrana se torne mais hidrofílica, característica essa que, associada aos poros formados, proporciona melhor desempenho quanto às rejeições ao soluto.

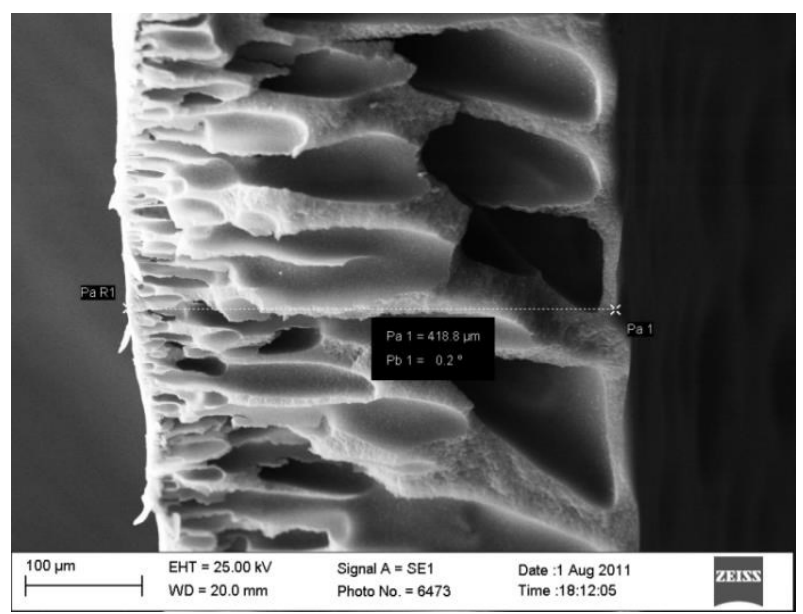

Figura 8: Seção transversal da membrana Mb2 (8,5\% de PVP).

Na Figura 9, fotomicrografia que expõe a superfície inferior da membrana Mb2, observa-se a presença de poros irregulares em termos de diâmetro e de geometria. Com o aumento da concentração em massa da PVP, o aditivo gera maior densidade porosa, aproximadamente 968 poros em uma área de $478,81 \mu \mathrm{m}^{2}$, sendo que o diâmetro médio aparente dos poros maiores é de aproximadamente $0,367 \pm 0,05 \mu \mathrm{m}$, e o diâmetro médio aparente dos poros menores é de aproximadamente $0,166 \pm 0,08 \mu \mathrm{m}$. O efeito provocado pela adição de maior massa da PVP na formação das membranas já foi observado por outros pesquisadores [33, 34 e 35], e esses relacionaram o aumento em massa da PVP com o aumento da quantidade de poros e a diminuição dos seus diâmetros em membranas de polietersulfona.

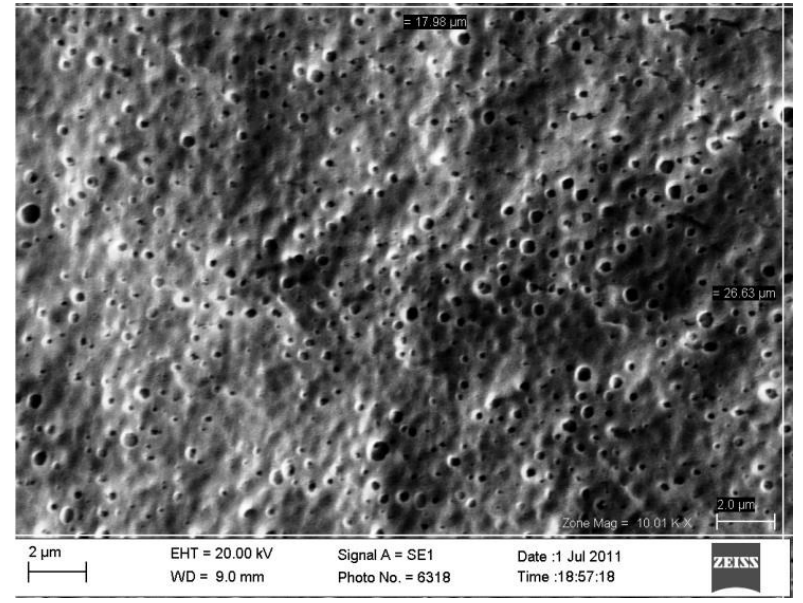

Figura 9: Superfície inferior da Mb2 (8,5\% de PVP). 


\section{CONCLUSÕES}

As análises por meio da técnica de IV-TF mostraram as principais bandas presentes no polímero base (PAN), bandas essas: em $2246 \mathrm{~cm}^{-1}$, associada à deformação axial do grupo $\mathrm{C} \equiv \mathrm{N}$ da unidade de acrilonitrila, em $1735 \mathrm{~cm}^{-1}$, associada ao estiramento do grupo carbonila $(\mathrm{C}=\mathrm{O})$, presente no monômero éster ou ácidos utilizados na polimerização da PAN; no copolímero - $\mathrm{P}(\mathrm{AN}-\mathrm{co}-\mathrm{AV})$ em $1235 \mathrm{~cm}^{-1}\left(v_{\mathrm{C}-0}\right)$, associada aos grupos acetatos presentes no copolímero e no aditivo polimérico PVP atribui-se seus comprimentos de onda em $1664 \mathrm{~cm}^{-1}$ e $1293 \mathrm{~cm}^{-1}$. Portanto, essa técnica é bastante sensível, e capaz de identificar até mesmo os resíduos remanescentes de PVP na membrana.

A fotomicrografia, obtida pela MEV, mostra que com o aumento na concentração de PVP (de 6,5\% para $8,5 \% \mathrm{~m} / \mathrm{m}$ ) promoveu alterações morfológicas positivas nas membranas tais como: presença de "pele" seletiva mais densa na superfície superior; supressão de espaços vazios; estiramento e reorganização dos canais porosos presentes na seção transversal, dando origem à forma cônica dos canais; e por fim, aumento da densidade porosa e diminuição do diâmetro dos poros (superfície inferior).

\section{BIBLIOGRAFIA}

[1] HABERT, A. C., BORGES, C. P., NÓBREGA, R. Processos de Separação por Membranas. Rio de Janeiro, E-papers, 2006.

[2] BRITO JÚNIOR, C. R., FLEMING, R., PARDINI, L. C., ALVES, N. P. "Análise térmica da poliacrilonitrila plastificada com glicerol em extrusora”, Polímeros: Ciência e Tecnologia, v. 22, n. 4, pp. 364-368, Ag. 2012.

[3] RARALINGAM, P. \& RADHAKRISHNAN, G. Polyacrylonitrile precursor for carbon fibers- Journal of Macromolecular Science, Part C: Polymer Reviews, v. 31, n 2-3, pp. 301-310, Maio 1991.

[4] RADOVANOVIC, P., THIEL, S.W., HWANG, S.T. "Formation of asymmetric polysulfone membranes by immersion precipitation. Part II. The effects of casting solution and gelation bath compositions on membrane structure and skin formation”, Journal of Membrane Science, v. 65, n.3, pp. 231-246, Janeiro 1992.

[5] STRATHMANN, H., SCHEIBLE, P., BACKER, R. W. "A rationale for the praparation of LoebSourirajan type cellulose acetate membrane", Journal of Applied Polymer Science, v. 15, n.4, pp. 811-828, Abril 1971.

[6] LAVALL, R. C., GARCIA, M. E., NOBREGA, R. "Preparação, Caracterização e Aplicação de Membranas Anisotrópicas de Poliacrilonitrila”, Polímeros Ciência e Tecnologia, v. 9, n. 4, p. 52-57, Dezembro 1999.

[7] NOUZAKI, K., NAGATA, M., ARAI, J., et al.,"Preparation of polyacrylonitrile ultrafiltration membranes for wastewater treatment", Desalination, v. 144, n. 1-4, pp. 53, Setembro 2002.

[8] BRITO JÚNIOR, C. A. R., FLEMING, R. R., PARDINI, L. C., et al. "Análise térmica da poliacrilonitrila plastificada com glicerol em extrusora", Polímeros: Ciência e Tecnologia, v. 22, n. 4 , pp. 364-368, Agosto 2012.

[9] KIM, I-C., YUN, H-G., LEE, K-H. "Preparation of Asymmetric Polyacrylonitrile membrane with small pore size by phase inversion and post-treatment process", Journal of Membrane Science, v. 199, n. 1-2, pp. 75-84, Abril 2002.

[10] MULDER, M. Basic Principles of Membrane Technology. Kluwer Academic Publishers, London, v. 109,n.12, pp. 1420-1421, Setembro 1996.

[11] JUNG, B. "Preparation of hydrophilic polyacrylonitrile blend membranes for ultrafiltration", Journal of Membrane Science, v. 229, n. 1-2, pp. 129-136, Fevereiro 2004.

[12] CAPANNELli, G., VIGO, F., MUNARI, S. "Ultrafiltration Membranes-Characterization Methods".,Journal of Membrane Science, v. 15, n. 3, pp. 289-313, Outubro 1983.

[13] KANG, J. S., LEE, Y. M. "Effects of Molecular Weight of Polyvinylpyrrolidone on Precipitation Kinetics During the Formation of Asymmetric Polyacrylonitrile Membrane", Wiley Periodicals, Inc. Journal of Applied Polymer Science, v. 85, n. 1, pp. 57-68, Julio 2002.

[14] YANAGISHITA, H., NAKAME, T., YOSHITOME, H. "Selection criteria for solvent and gelation medium in the phase inversion process", Journal Membrane Science, v. 89, n. 3, pp. 215, Abril 1994. 
[15] MATHUR, R. B., BAHL, O. P., SIRVAN, P. "Thermal degradation of polyacrylonitrile fibers", Current Science, v. 62, n. 10, pp. 662-669, Maio 1992.

[16] FLEMING, R. R. Caracterização de Fibras e Filmes de Poliacrilonitrila Extrudada, Dissertação M.Sc, ITA, São José dos Campos, SJC, Brasil, Maio 2010.

[17] WAN, L. S., XU, Z. K., HUANG, X. J. “Asymmetric membranes fabricated from poly (acrylonitrile-coN-vinyl-2-pyrrolidone) with excellent biocompatibility”, Journal of Polymer Science, v.102, n. 5, pp.45774583, Setembro 2006.

[18] SUI, X. et al. "Structural and photoluminescent properties of $\mathrm{ZnO}$ hexagonal nanoprisms synthesized by microemulsion with polyvinyl pyrrolidone served as surfactant and passivant", Chemical Physics Letters, v.424, n. 4-6, pp. 340-344, Junho 2006.

[19] WAN, L. S. et al. "Copolymerization of acrylonitrile with N-vinyl-2-pyrrolidone to improve the hemocompatibility of polyacrylonitrile”, Polymer, v.46, n. 18, pp.7715-7723, Agosto 2005.

[20] ZHANG, Z. et al. "Polyacrylonitrile and carbon nanofibers with controllable nanoporous structures by electrospinning”, MacromolecularMaterials and Engineering, v. 294, n, 10, pp.673-678, Outubro 2009.

[21] DUMITRAŞCU, M. et al. "Characterization of electron beam irradiated collagen polyvinylpyrrolidone (PVP) and collagen-dextran (DEX) blends", Digest Journal of Nanomaterials and Biostructures, v.6, n.4, p.1793-1803, 2011.

[22] STRATHMANN, H., KOCK, K., AMAR, P, et al. "The formation mechanism of asymmetric membranes", Desalination, v.16, n. 2, pp.179-203, Abril 1975.

[23] PORTER, M. C. Handbook of Industrial Membrane Technology., New Jersey, Noyes Publication, 1990.

[24] WIENK, I. M. et al. "Recent advances in the formation of phase inversion membrane made from amorphous or semi-crystalline polymers", Journal of Membrane Science, v.113, n. 2, pp.361-371, Maio 1996.

[25] MACHADO, P. S. T., HABERT, A. C., BORGES, C. "Membrane formation mechanism based on precipitation kinetics and membrane morphology: flat and hollow fiber polysulfone membranes", Journal of Membrane Science, v.155, n. 2, pp.171-183, Abril 1999.

[26] VAN DE WITTE, P., DIKJKSTRA, P.J., VAN DEN BERG, J. W. A., et al., "Phase separation processes in polymer solution in relation to membrane formation", Journal of Membrane Science, v. 117, n. 1-2, pp. 1-31, Março 1996.

[27] CARVALHO, R. B., BORGES, C. P., NÓBREGA, R. "Formação de membranas planas celulósicas por espalhamento duplo para os processos de nanofiltraçao e osmose inversa", Polímeros: ciência e tecnologia, v.11, n.2, p.65-75, Junho 2001.

[28] BOOM, R. M.; BOOMGARD, T. V. D.; SMOLDERS, C.A. "Mass transfer and thermodynamic during immersion precipitation for a two-polymer system: evaluation with the system PES-PVP-NMP-wather". Journal of Membrane Science, v.90, n. 3, pp. 231-249, Maio 1994.

[29] CHUANG, W. Y., et al. "The effect of polymeric additive on the structure and permeability of poly (vinyl alcohol) asymmetric membranes”. Polymer, v.41, n. 15, pp.5633-5641, Julho 2000.

[30] WIENK, I. M. et al. "Chemical treatment of membranes of a polymer blend: mechanism of the reaction of hypochlorite with poly (vinyl pyrrolidone)”, Journal Polymer Science, v.33, n. 1, pp.49, Janeiro 1995.

[31] YUAN, Z., LI, X. D. "Porous PVDF/TPU blends asymmetric hollow fiber membranes prepared with the use of hydrophilic additive PVP (K30)”, Desalination, v.223, n. 1, pp. 438-447, Março 2008.

[32] JUNG, B. et al. "Effect of molecular weight of polymeric additives on formation, permeation properties and hypochlorite treatment of asymmetric polyacrylonitrile membranes", Journal of Membrane Science, v.243, n. 1-2, pp.45-57, Novembro 2004.

[33] LAFRENIERE, L. Y. et al. "Effect of polyvinylpyrrolidone additive on the performance of polyethersulfone ultrafiltration membranes”, Industrial \& Engineering Chemistry Research, v.26, n. 11, pp.2385-2389, 1987.

[34] BOOM, R. M. et al. "Microstructures in phase inversion membranes - part 2: the role of a polymeric additive”, Journal of Membrane Science, v.73, n. 2-3, pp.277-292, Abril 1992. 
FARIA, P.C.; MARTIN, A.A.; ALVES, N. P. revista Matéria, v.22, n.1, 2017.

[35] MARCHESE, J. et al. "Fouling behavior of polyethersulfone UF membranes made with different PVP", Journal of Membrane Science, v.211, n. 1, pp.1, Janeiro 2003. 